\title{
Un taller literario y un portal para divulgar la ciencia: Cienciorama
}

\author{
Guadalupe Zamarrón Garza, \\ Alicia García Bergua y \\ Luci Cruz Wilson
}

\section{Resumen}

El Taller/Portal Cienciorama es un espacio para escribir de manera creativa sobre ciencia. En él participan estudiantes de licenciatura, maestría y doctorado en ciencias naturales, exactas, sociales y humanidades de todo el país, pero principalmente de la UNAM. En el taller se crean artículos y noticias que se publican en el portal Cienciorama. Los materiales tratan investigaciones recientes o temas vistos desde una perspectiva actual que refleja la continua búsqueda y perpetua construcción del conocimiento científico. El Taller se lleva a cabo en línea y de manera presencial; esto último lo convierte también en un espacio de diálogo muy importante para propiciar una cultura científica en español. Con el taller se busca que los participantes desarrollen herramientas literarias para trasladar el conocimiento científico al público general con énfasis en el universitario, interesados en conocer el estado actual de la ciencia; esto con el fin de promover el diálogo y el intercambio de ideas entre ellos que fortalezcan su nivel educativo en ciencia.

Palabras clave: taller, portal, internet, divulgación, conocimiento, ciencia, lenguaje, creatividad, cultura, libertad, democracia.

\section{A literary workshop and a website to popularize science: Cienciorama}

\begin{abstract}
The Workshop/Portal Cienciorama is a space for writing about science creatively; the participants in this workshop are graduate and undergraduate students from all the country but mainly from UNAM working on exact, natural, and social sciences, and humanities. The articles and news produced in the workshop are published on Cienciorama's website. The materials address recent studies or issues viewed from a contemporary perspective that reflects the continuous search and perpetual construction of scientific knowledge. The workshop takes place online and in face-to-face meetings; because of this, the workshop is an important space of dialogue as well, generating a culture of science in Spanish. The workshop aims to help the participants develop the literary tools required to translate scientific knowledge to a general public with emphasis in university audience that is not specialized in science in order to support the dialogue and exchange of ideas among them and strengthen theirs educational science level.
\end{abstract}

Key words: workshop, portal, internet, science popularization, knowledge, science, language, creativity, culture, freedom, democracy.

DOI: http://doi.org/10.22201/codeic.16076079e.2018.v19n2.a9 


\section{Guadalupe Zamarrón Garza}

Tiene estudios de física, pintura, música y realización cinematográfica en el Centro de Capacitación Cinematográfica en la UNAM. Desde el 2001 colabora en el Proyecto Cienciorama. Ha desarrollado principalmente en dos vertientes: editorial y audiovisual. Editorial: en las revistas Naturaleza y Chispa, principalmente. Coedición de libros sobre comunicación pública de la ciencia con universidades del país, cubanas, brasileñas y españolas. Audiovisual: Conducción y realización de la serie La Ciencia y Usted transmitida por canal 11, y programas documentales por Canal 4, ambos productos del Conacyt. Realización de películas en 16 mm de la AIC (HOY AMC) y audiovisuales sobre educación en la SEP. Estudio sobre el sistema mexicano de educación superior para la ANUIES. Miembro fundador de la Somedicyt y presidenta entre 1991-2. Gestora de los congresos nacionales de divulgación de la ciencia, organizadora del congreso I y II y del Premio Alejandra Jaidar organizados por la Somedicyt. Organizadora del EventoCurso de Ciencias Planetarias con el Museo de la CFE y Somedicyt: pláticas de divulgación impartidas por el Físico Luis Álvarez, presentación y diálogo en los Institutos de Astronomía y Geofísica de la UNAM y el curso y las pláticas en el Museo de la CFE. Iniciadora del área de televisión del Conacyt. 1973. Labores técnico-académicas en CUPRA, CUCC, TV y la DGIA, de la UNAM. Actualmente (2017-18) es coordinadora del Proyecto Cienciorama que alberga la Dirección General de Divulgación de la Ciencia de la UNAM y auspicia la Sociedad Mexicana para la Divulgación de la Ciencia, SOMEDICYT.

\section{Alicia García Bergua}

Es escritora. Nació en la Ciudad de México el 9 de septiembre de 1954. Estudió la licenciatura de filosofía en la Facultad de Filosofía y Letras de la Universidad Nacional Autónoma de México. De 1975 a 1988 trabajó en la revista Naturaleza, de la que llegó a ser jefa de redacción, y en el Centro de Comunicación de la Ciencia de la UNAM, donde se formó para hacer divulgación científica con el grupo fundador de la divulgación científica en México dirigido por Luis Estrada. Fue editora en la primera etapa como revista del suplemento La Jornada Semanal, 1988-1991. Coordinadora de publicaciones del Instituto Nacional Indigenista, 1991-1993. Guionista y asistente de la Coordinación de Información del Noticiero Cultural 9:30 de Canal 22 desde su comienzo en 1993 hasta 1996. Editora de la revista Este País de 1996 a 2001 y asistente editorial de Scientific American- México de 2004 a 2006. Ha realizado gran cantidad de trabajo editorial y de traducción de textos especializados y de divulgación de la ciencia, además de algunos libros. Ha impartido cursos y talleres sobre poesía en la Universidad del Claustro de Sor Juana (2009 y 2010) y en la Sociedad General de Escritores de México (SOGEM) (2008 y 2017). Trabaja actualmente en la Dirección General de Divulgación de la Ciencia de la UNAM como editora e instructora del taller-portal de escritura creativa en divulgación científica Cienciorama, y como editora y escritora de textos de divulgación de la ciencia en la revista de ciencia para jóvenes ¿Cómo ves? Es autora de los libros de poesía Fatigarse entre fantasmas (Ediciones Toledo, 1991), La anchura de la calle (Conaculta, col. Práctica Mortal, 1996), Una naranja en medio de la tarde (Libros del Umbral/ Pablo Boullosa, 2005); Tramas (Cálamos-INBA-Conaculta, 2007), El libro de Carlos (Ed. Juan Malasuerte, 2007) y Ser y seguir siendo (editorial Textofilia 2013), del libro de ensayos Inmersiones (Dirección General de Publicaciones, UNAM, 2009) y de un libro de ensayos aún inédito La lucha con la zozobra. La libertad bajo palabra en México de cuatro poetas modernos: Villaurrutia, Owen, Cuesta y Paz y un libro de poemas inédito aún también, Salto y sueño. Ha sido miembro del Sistema Nacional de Creadores de México en los periodos 2001-2007, 2011-2013 y lo es actualmente.

\section{Luci Cruz Wilson}

Estudió biología en la Facultad de Ciencias de la UNAM. En 1982 se integró al Centro Universitario de Comunicación de la Ciencia de la UNAM dirigido por Luis Estrada. Como divulgadora de la ciencia ha trabajado en proyectos editoriales como autora o coautora de 28 libros de texto, de divulgación científica y diseño de juegos para niños (CONAFE, CONACULTA/ADN Editores, Santillana, Nuevo México, DGDC, ACMor, SEP). Ha ideado y realizado la coordinación editorial de las colecciones de libros para niños Salud 
para todos (ADN Editores/Instituto de Ciencia y Tecnología del DF) y ¿Qué te comes? (Academia de Ciencias de Morelos). Fue editora del boletín Salvia (INSP) y de la revista Pronatura. Ha participado en 23 proyectos museográficos, en las áreas de planeación, investigación documental e iconográfica (Margen Rojo, A.C.; Concepto M; Somedicyt), algunos de ellos son: Expo Lisboa -Exposición temporal- Entre Mares (1998); Museo de Historia Natural TAMUX, Cd. Victoria, Tamaulipas (2004); Exposición temporal Hábitat del Riesgo a la Sustentabilidad, Forum Monterrey (2007); Museo Centro de Interpretación Ecológica, Reserva de la Biósfera El Cielo, Tamaulipas (2010); Exposición temporal UNAM, Premio Príncipe de Asturias, Toledo, España (2010); Exposición temporal Paseo en Mapa, D.F. Antiguo Colegio de San Ildefonso (2010). Proyecto de remodelación, museografía y actualización de contenidos del Museo de Historia Natural de la Ciudad de México (2013). Ha participado en proyectos de televisión y video como guionista en series sobre ciencia y medio ambiente (TVUNAM, Filmoteca de la UNAM, Canal 11 y Canal 22). Fue Coordinadora general del programa de televisión Ciencia Hoy (TVUNAM/Canal 11). Desde 2012 participa como Coordinadora del Taller/Portal Cienciorama de la DGDC-UNAM.

\section{Los cimientos}

Como antecedente de Cienciorama está el trabajo que se llevaba a cabo en el Centro Universitario de Comunicación de la Ciencia (CUCC) de la UNAM, creado en 1980 por Luis Estrada Martínez, fundador de la divulgación científica en México e institucionalizado por el rector Pablo González Casanova. Ahí se realizaban dos importantes publicaciones para la divulgación científica en México, la revista Naturaleza y el boletín Prenci, además de otras actividades a través de distintos medios y dirigidas a diferentes públicos. En todas ellas se consideraba fundamental que se hablara y pensara sobre ciencia en español, ya que ésta no era —ni lo es actualmente- la lengua básica en el estudio de las carreras, ni en gran parte de la investigación científicas; por lo tanto, reflexionar sobre el uso adecuado del español se volvió un tema de la divulgación. Luis Estrada pensaba que entre las cualidades indispensables de una buena divulgación está la claridad del mensaje y la fidelidad al conocimiento que se transmite con él. Como el lenguaje científico es muy especializado y el contexto en el que se genera es poco conocido, quienes hacen divulgación tienen que recurrir a herramientas literarias como metáforas y analogías para acercar al público a la ciencia, pero para mantenerse fieles a lo que comunican es muy importante el uso de un lenguaje preciso y ajustado a lo que se quiere transmitir y para ello se debe tener domino del lenguaje común. Además, el hecho de hablar y reflexionar sobre ciencia en ámbitos no académicos, en los que participan personas de distintas disciplinas, contribuye a que la ciencia, que en sí misma 
es cultura, esté presente en espacios más amplios y así propiciar diálogos que enriquezcan tanto el quehacer de los científicos como el de otras personas no directamente involucradas con la ciencia.

El desarrollo tecnológico y científico del siglo XX, que implicó una considerable matematización del conocimiento, impidió que los científicos describieran su trabajo de una manera literaria, tal como Maxwell y Darwin lo hicieron en el siglo XIX. En sus escritos, como bien lo señala Carlos López Beltrán —quien trabajó en el CUCC y formó parte del gran esfuerzo de la divulgación científica en México - había una gran voluntad de comunicación y un gran esfuerzo por expresarse. Muchos científicos del siglo XX abandonaron las tareas literarias y quienes las continuaron, por ejemplo Jared Diamond y Stephen J. Gould, se consideran excepcionales. No obstante, cada vez hay más científicos empeñados en escribir sobre sus conocimientos para un público general, pues ellos mismos

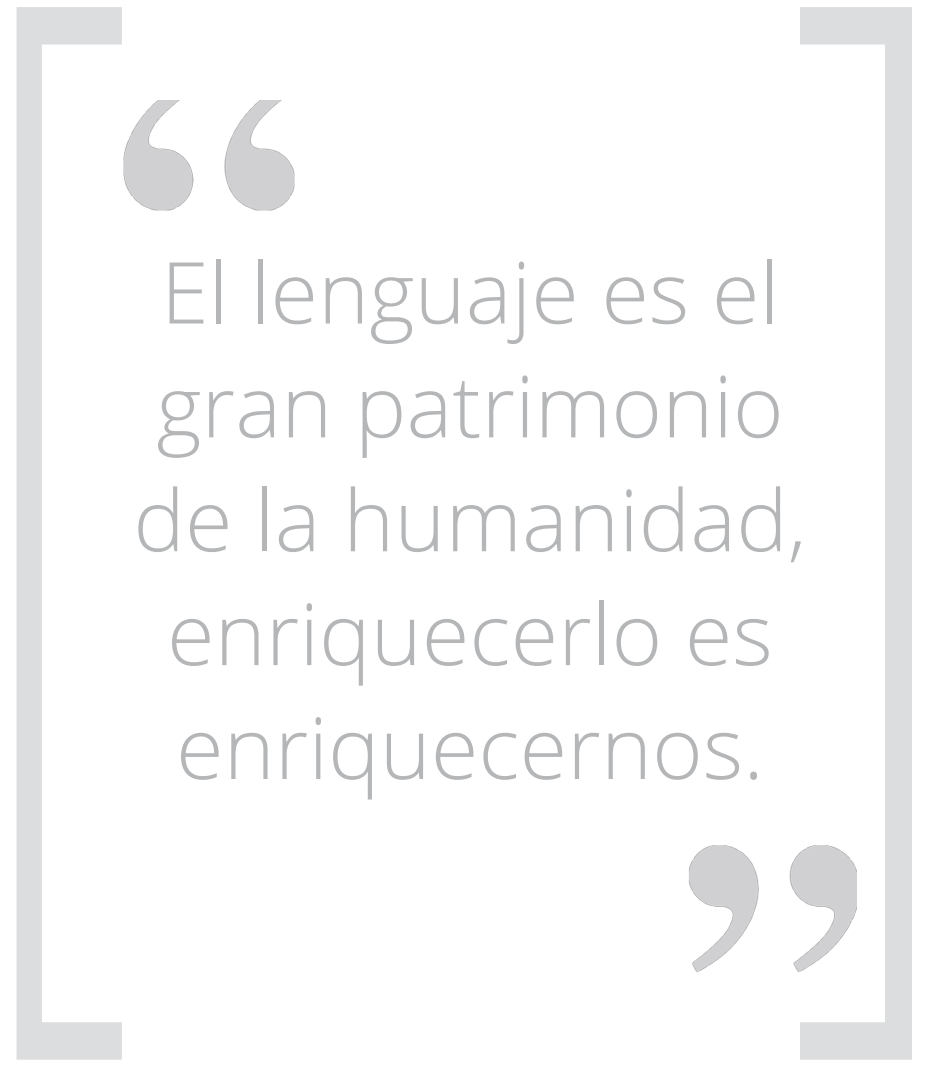
reconocen también la utilidad de ponerlo en ese horizonte más amplio del lenguaje que todos compartimos.

En 2002 Luis Estrada concibió el proyecto Temas de Ciencia Contemporánea, y como parte de éste, el portal Cienciorama que se mantuvo como taller digital por cerca de diez años. En ambos espacios se reanudaron parte de las actividades de divulgación de la ciencia emprendidas en el CUCC. A Cienciorama se subieron textos trabajados sobre los avances en las distintas ramas de la ciencia, y se dio cuenta de la interdisciplinariedad de la investigación científica y de los difusos límites entre cada una de sus ramas. Se trataba de dar un panorama viviente y dinámico del conocimiento científico en perpetua construcción. Estrada propuso agrupar los textos por la parte del universo con la que trataban y no por disciplinas académicas. Todo esto dirigido al público universitario y más allá, como parte del proyecto Temas de Ciencia Contemporánea se invitó a distintos investigadores de diversas instituciones a hablar de sus investigaciones, de lo más reciente en su campo y de su proyección hacia el futuro.

\section{De una cafetería a la Teleaula Luis Estrada de Universum}

A finales del 2012 Cienciorama convocó a un taller presencial al que respondieron estudiantes de licenciatura, maestría y doctorado de distintas especialidades científicas que tenían en mente la idea de aprender a expresar sus ideas y 
compartir sus conocimientos. Más tarde se unieron estudiantes de economía, sicología, historia, filosofía y más científicos. De hecho, el afluente de nuevos colaboradores ha sido variable pero continuo. El formato del portal también cambió como respuesta a las actividades del taller pues la estructura, la fluidez y el diseño debían corresponder con su dinámica. Se cambiaron la imagen y la estructura del portal para que fueran amigables y sencillas; se agregó un nuevo buscador, se permitió la lectura directa de los textos y la posibilidad de bajarlos en formato PDF; se facilitó la descarga y visualización desde cualquier dispositivo y especialmente su difusión en redes sociales. Se buscó una programación computacional con lenguajes y códigos actuales y adaptables para asegurar que el sitio tuviera una vida óptima cercana a los ocho años.

Para 2013 y con el apoyo de la Sociedad Mexicana para la Divulgación de la Ciencia y la Técnica (SOMEDICyT), el equipo del nuevo Taller-Portal Cienciorama se consolidó y cambió la sede de la plataforma a la Dirección General de Divulgación de la Ciencia (DGDC). Por su parte, los talleres presenciales pasaron de tener lugar en diversas cafeterías del entorno de la Ciudad Universitaria a las instalaciones de la Teleaula Luis Estrada de Universum. El Portal cambió de imagen, sin embargo, el espíritu siguió siendo el mismo: el trabajo interdisciplinario y la discusión en libertad.

\section{Elementos que conforman}
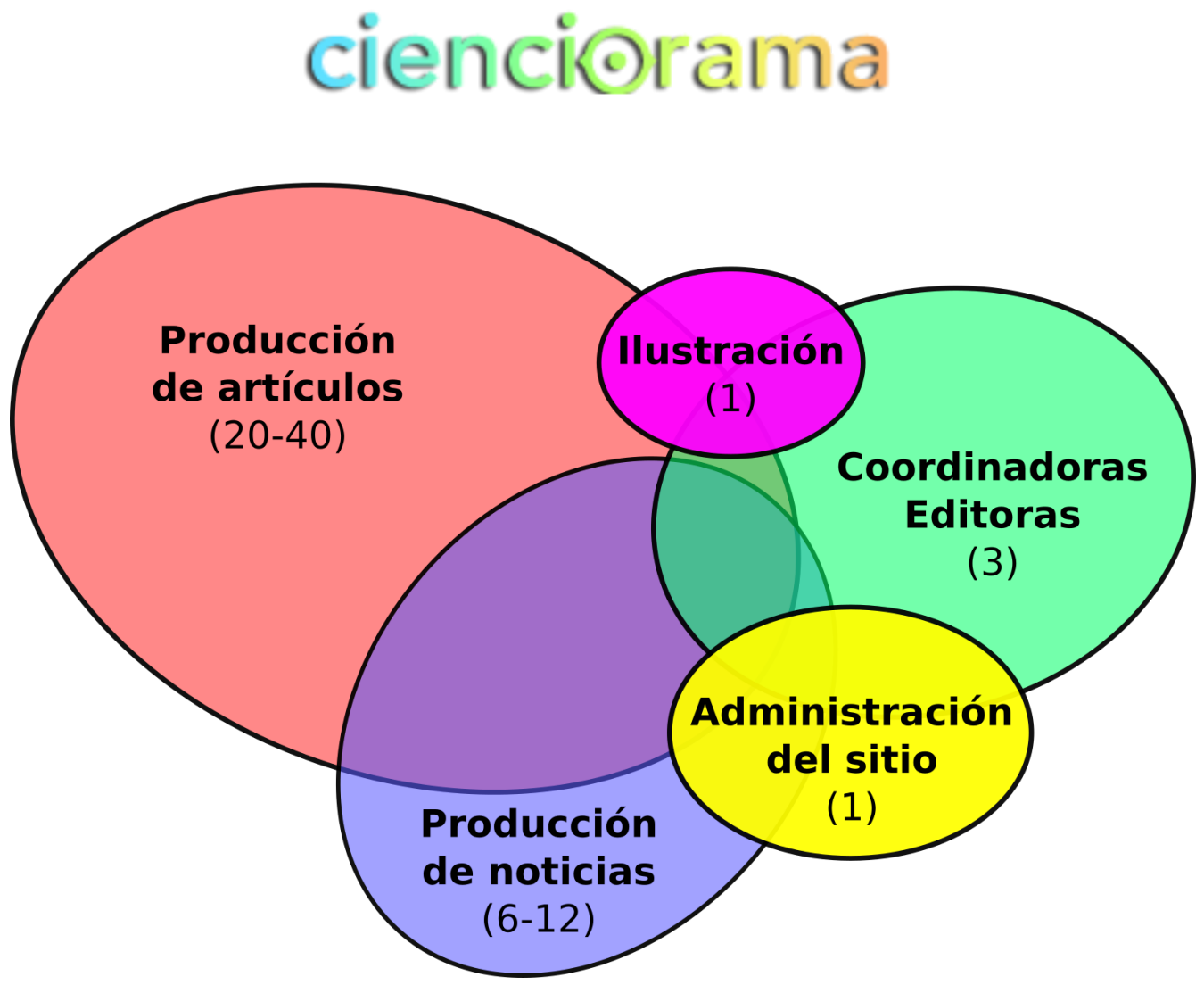


\section{Un taller-un medio}

Cienciorama consiste en un taller presencial y en línea donde se elaboran artículos y noticias, así como un portal que lleva el mismo nombre donde se publican los materiales producidos en el taller. Lo conducimos las autoras de este texto, un administrador del portal y redes sociales, una ilustradora eventual —ambos surgidos de entre los participantes del taller - y un número variable de estudiantes colaboradores. Ha funcionado ininterrumpidamente por más de cinco años y no existe un currículum que los participantes deban cubrir.

El taller se realiza de manera presencial cada dos semanas; ahí los colaboradores presentan los temas que quieren tratar según sus intereses y desempeño en distintas áreas científicas. Una vez planteado el tema que cada participante eligió, surgen preguntas, dudas y comentarios. Esto da pie a discusiones e intercambios de información. Una vez definido el tema y sus alcances puntuales, los colaboradores toman lo que les sirve para armar su texto. Posteriormente realizan un esquema en el que definen lo que quieren comunicar y desglosan los subtemas necesarios para lograrlo considerando ciertos lineamientos editoriales.

\section{Procesamiento de artículos en el Taller/Portal}
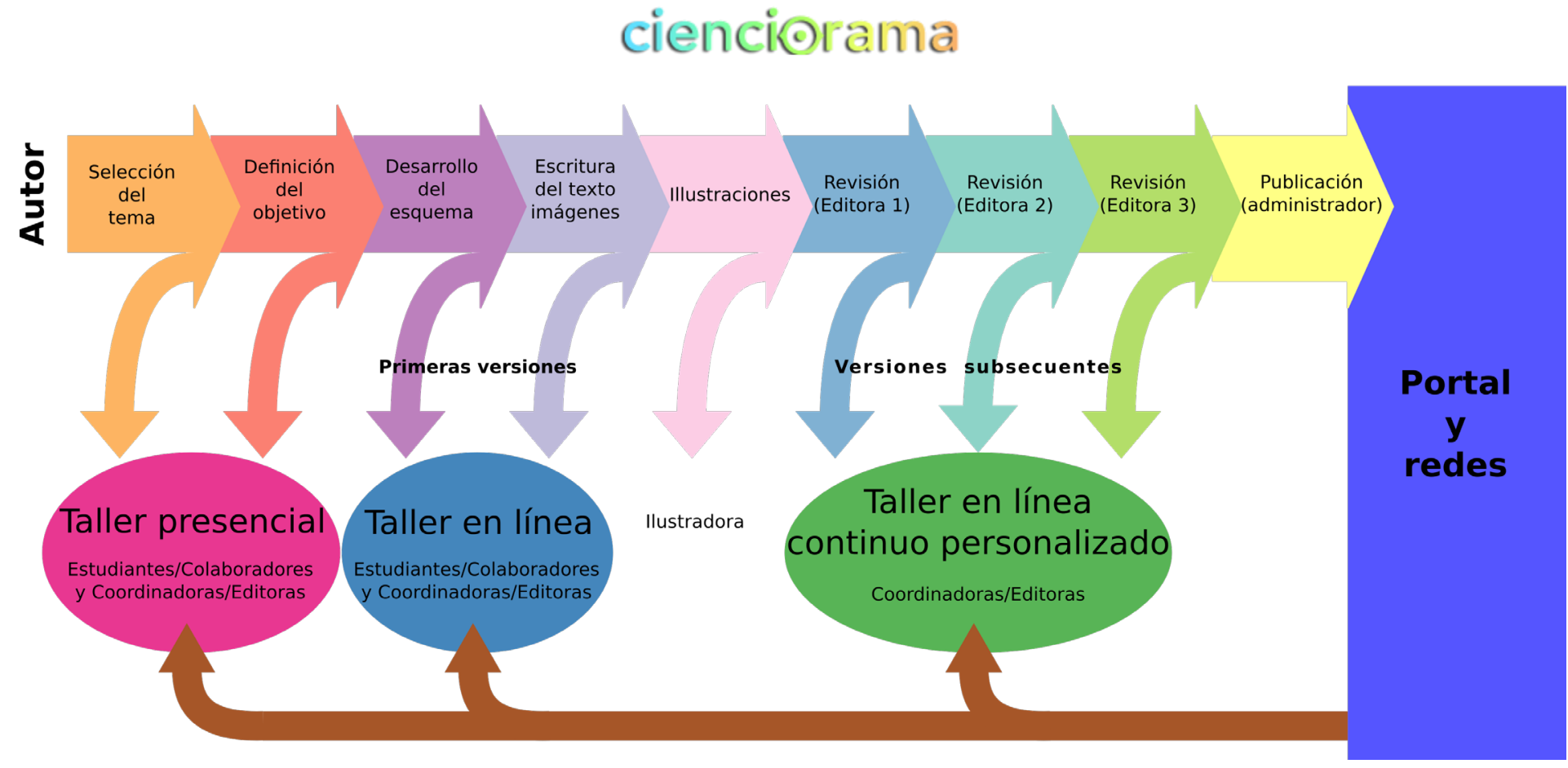

En el formato en línea del taller, cada artículo va y viene entre las tres editoras y el autor hasta lograr una versión final. El proceso tiene una duración variable dependiendo de la disposición de tiempo de quien escribe, del manejo del lenguaje, del contenido y de la selección y adaptación de imágenes o la 
confección ex profeso de éstas. Una vez concluido el artículo queda en espera para su publicación en la sección más adecuada del portal que tiene una clasificación no académica del conocimiento científico: de sociedad y tecnología, la vida, la tierra, ciencia en el espejo, el organismo humano, micro y macrocosmos.

\section{Procesamiento de noticias en el Taller/Portal cienci०)}

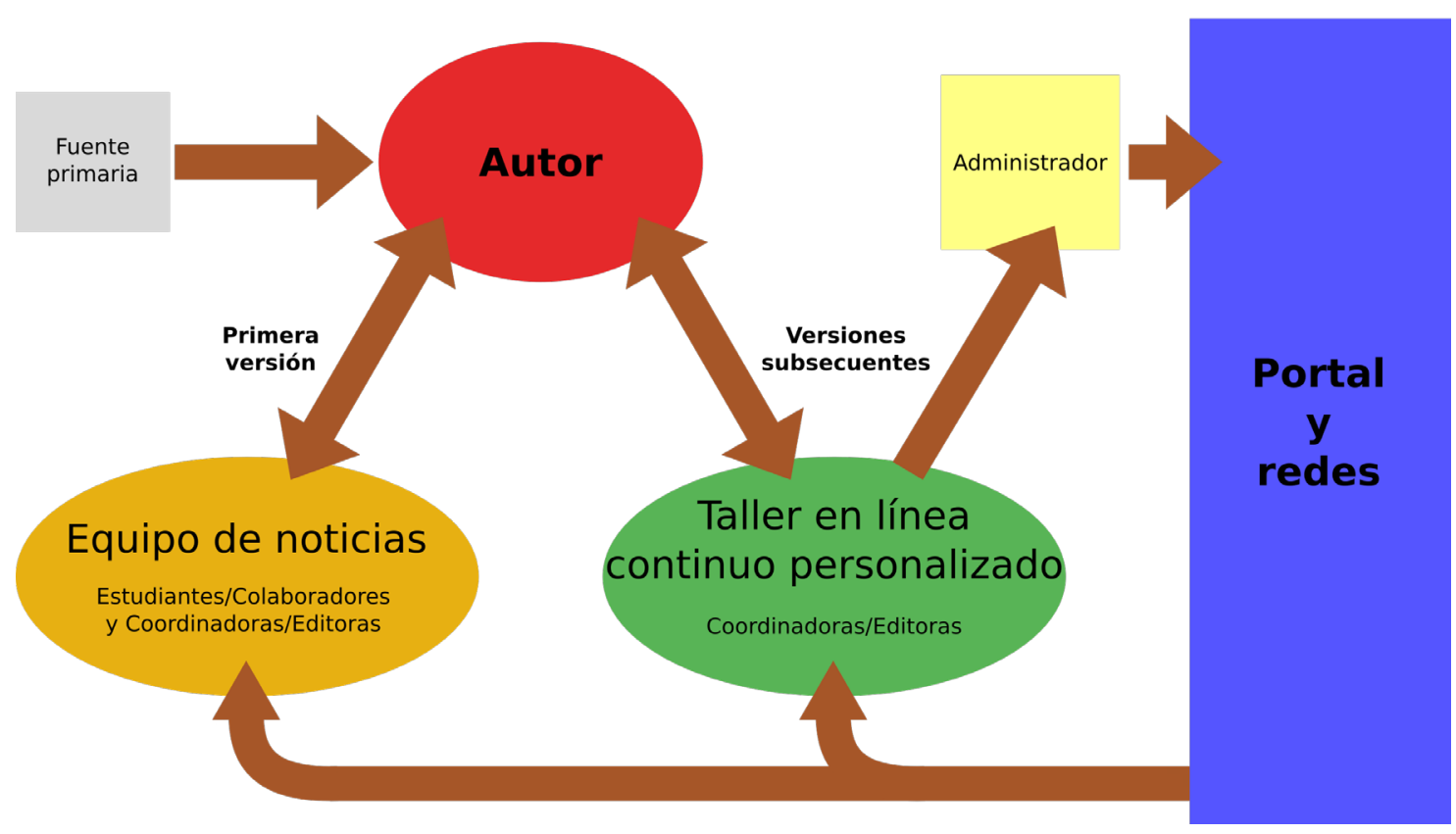

\section{Ampliar horizontes}

En algunos talleres presenciales también participan científicos y otros profesionales de la divulgación científica a quienes se les invita a platicar sobre sus trabajos. Por ejemplo: Carlos López Beltrán (investigador y divulgador del Instituto de Filosofía/UNAM) habló del uso de metáforas para divulgar la ciencia; Miguel Ángel Cevallos (investigador y divulgador del Centro de Ciencias Genómicas/UNAM) habló de su trabajo como divulgador y científico y señaló la importancia de dar a conocer aquello que a uno le apasiona, así como no dudar de la inteligencia de los lectores; Sergio de Régules (divulgador de la DGDC/UNAM) puntualizó que el público no está cautivo, que hay que seducirlo y una de las herramientas es el humor; Estrella Burgos (editora de la revista ¿Cómo ves? DGDC/UNAM) en su charla sobre periodismo científico, insistió en que éste debe ser neutral, objetivo y que sirva y haga valer su importancia en la libertad de los ciudadanos; Agustín López Munguía (investigador y divulgador del Instituto de Biotecnología/UNAM) abordó el tema de los Organismos Genéticamente Modificados y lo que la prensa publica sobre éstos; Alicia Castillo (investigadora y divulgadora del Instituto 
de Investigaciones en Ecosistemas y Sustentabilidad/UNAM) nos compartió su experiencia en el quehacer de la divulgación en medios rurales; Luis Felipe Rodríguez (investigador y divulgador del Instituto de Radioastronomía y Astrofísica/ UNAM Morelia) abundó sobre la observación de planetas en otras estrellas; Antonio Lazcano (profesor y divulgador de la Facultad de Ciencias/UNAM) dialogó con el taller sobre el material hereditario, la historia de un debate entre la física y la biología; Rubén Barrera (investigador y divulgador del Instituto de Física/ UNAM) dio una cátedra sobre cómo presentar una plática de divulgación utilizando el tema de la posible invisibilidad de algunos materiales. Por otro lado, la ilustradora Eva Lobatón (ilustradora) asistió al taller para compartir su experiencia acerca de cómo interpretar una idea científica a través de una ilustración; Armando Arellano Ferro (investigador del Instituto de Astonomía/UNAM) conversó sobre los cúmulos globulares que consisten en cientos de miles de estrellas unidas gravitacionalmente. Éstos y otros especialistas nos han compartido saberes y han sido de gran valor para el equipo de colaboradores de Cienciorama.

\section{Cienciorama desde otras ventanas}

Otros esfuerzos por comunicar y hacer llegar la información científica a un público más amplio aprovechan el acervo del Taller/Portal Cienciorama. El Portal forma parte de la página de Ciencia UNAM. La Academia de Ciencias de Morelos ha reproducido y publicado en su página y en la columna de La Unión de Morelos muchos textos creados en el taller. Por su parte, la agencia Investigación y Desarrollo publica y difunde nuestros textos en su página. La revista ¿Cómo ves? También ha retomado algunos trabajos originales de Cienciorama y los ha adaptado a sus líneas editoriales. Asimismo SOMEDICyT presenta el portal Cienciorama entre sus proyectos.

\section{Peras y manzanas}

Los frutos del Taller/Portal Cienciorama son artículos y noticias. Los primeros tienen una estructura sencilla: un resumen seguido de varios apartados para hacer la lectura pausada y amable, y en ellos se busca dar luz sobre temas muy diversos, por ejemplo El Misterio del polvo, o la Guía teórica para un nocaut infalible, o la interrogante de si somos los únicos seres vivos en el universo; también es posible encontrar historias de amor y traición o los avances sobre la interfaz cerebro-máquina, entre muchas narraciones más.

Cienciorama incluye todos los género en sus textos; artículos descriptivos, ensayos, recuentos históricos, reportajes, narraciones con ficción o sin ella, entrevistas, crónicas y reseñas de libros. El Portal tiene ya un acervo de más de 500 artículos, y alrededor de 280 se han escrito desde 2013 como resultado 
del taller presencial. La sección de noticias se inició en enero de 2014 y dada la premura con la que tienen que subirse al sitio, sólo se trabajan en línea. A la fecha hay en el portal más de 600 noticias hechas bajo las premisas de responder el ¿qué?, ¿quién?, ¿dónde?, ¿cómo? y ¿cuándo? se dan. Muchas de ellas están aderezadas con entrevistas a los investigadores que produjeron la información y siempre están basadas en las fuentes primarias. Procuramos que los integrantes del equipo de noticias se especialicen en detectar información relevante de áreas de su interés como estudiantes de ciencias o disciplinas afines.

\section{Área y nivel de estudio de los miembros de}

\section{cienciorama}

- astrofísica

ciencias de la tierra
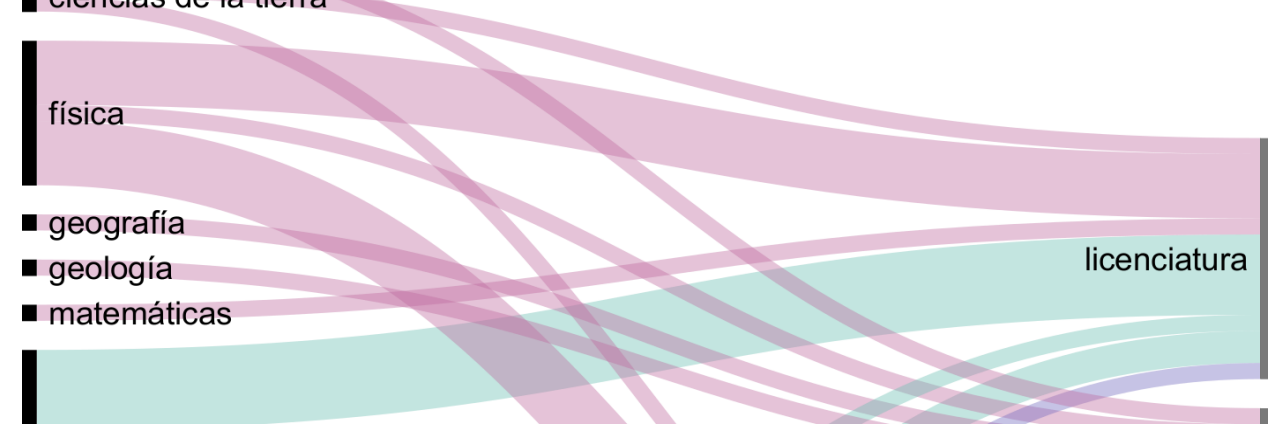

biología

maestría

biomédicas

genómicas

- ingeniería biomédica

doctorado

química

- química farmacobióloga

- abogado

- economía

posdoc

- escritor

filosofía

- letras inglesas

Dsicología 


\section{Para qué un taller de escritura creativa en ciencia}

Muchas universidades del mundo tienen talleres de escritura (ver referencias) para estudiantes graduados en ciencias pues esto no sólo redunda en que se haga divulgación de la científica, sino que implica también que los investigadores mejoren el planteamiento de proyectos, su presentación en diversos contextos y la exposición de contenidos, ideas y reflexiones en publicaciones científicas especializadas en cualquier foro que implique la palabra escrita.

En el Taller/Portal Cienciorama se reflexiona en grupo sobre conceptos, métodos, modelos y entornos del quehacer científico. En éste han participado alrededor de 130 estudiantes, aunque no todos han logrado publicar textos.

Del 2012 a la fecha se han publicado escritos de alrededor de 60 autores, entre artículos y noticias.

\section{Áreas que conforman cienciorama}

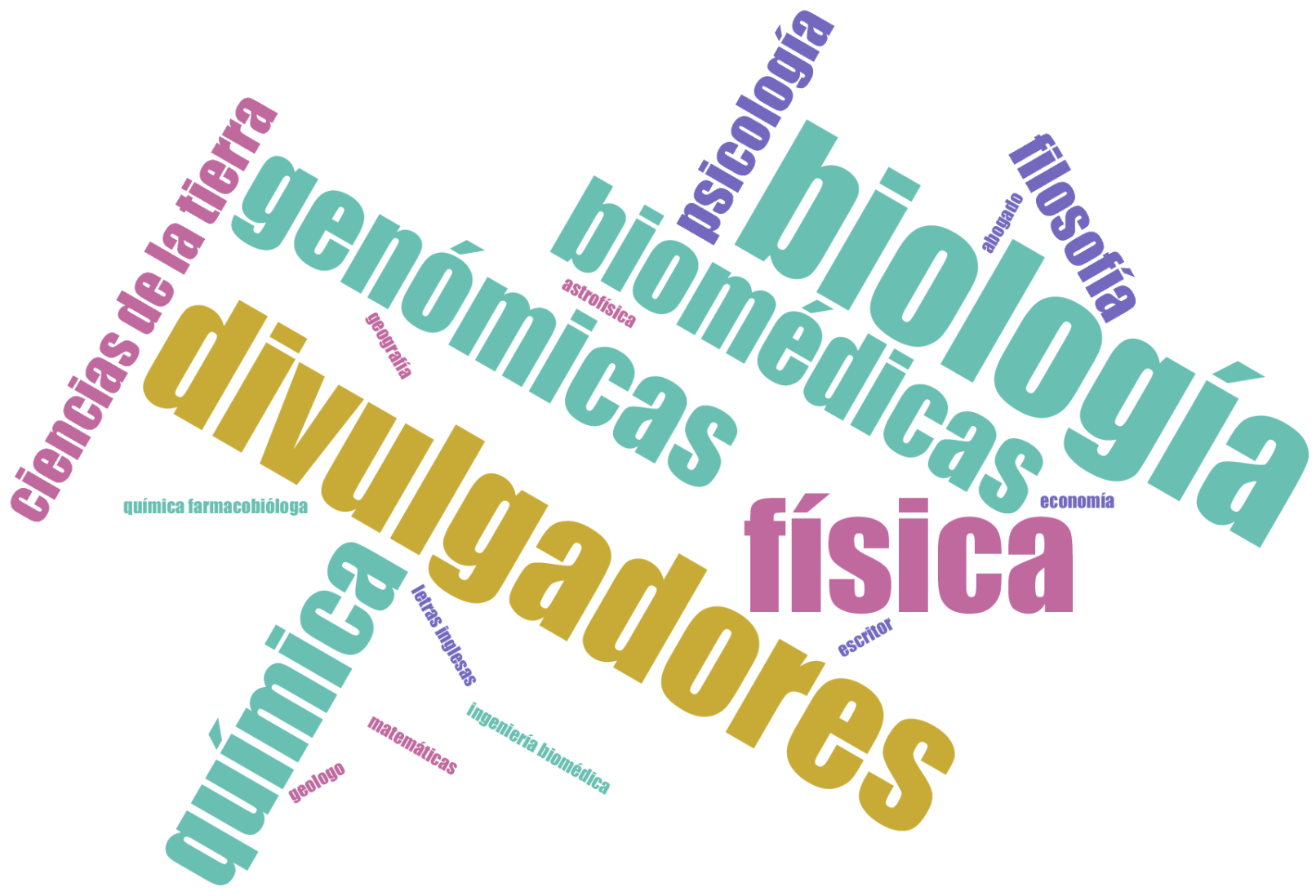




\section{Como la ciencia, Cienciorama está en constante construcción}

Por la característica dual de Cienciorama, nuestro proyecto debe ser evaluado desde la perspectiva de la formación de estudiantes en escritura creativa en ciencia y desde la del uso de un medio de comunicación; en este caso en concreto hablamos del internet que ha tenido en México un crecimiento exponencial y que en 2017 sobrepasó los 70 millones de usuarios, de acuerdo al INEGI. En cuanto a la parte de la evaluación, en este mismo número de la RDU hay un artículo que plantea los puntos de vista de algunos colaboradores. Por otro lado, hay en la red varias maneras de evaluar y analizar nuestro trabajo, desde Google Analytics, hasta los promedios de alcance diario en Facebook, sin embargo muchos de los índices expresados en las redes sociales están dirigidos al mundo de las ventas de productos (lo cual no implica que no nos funcionen ya que lo que hacemos, de alguna manera son productos culturales); para complementar quisimos interactuar más directamente con nuestros lectores a través de la aplicación de dos cuestionarios que pusimos en el portal durante dos periodos (diciembre 2014-marzo 2015 y los mismos meses en 2015-2016). En dichos cuestionarios solicitamos a los usuarios su opinión sobre la calidad de la información, el nivel de aceptación y la percepción de los objetivos, así como las características generales del portal: diseño, navegación, amigabilidad, etcétera.

Con base en los resultados de las encuestas - que debemos reconocer no son concluyentes - y recurriendo al sentido común y a la autocrítica, trabajamos en una reestructuración y diseño para simplificar la navegación, dar un localizador (URL) a la sección de noticias, modificar la sección de comentarios para que lleguen directamente a los autores e implementar alertas para alcanzar directamente a los usuarios que lo deseen, entre otras cosas.

\section{Artículos y noticias publicados por año y sección en el portal}
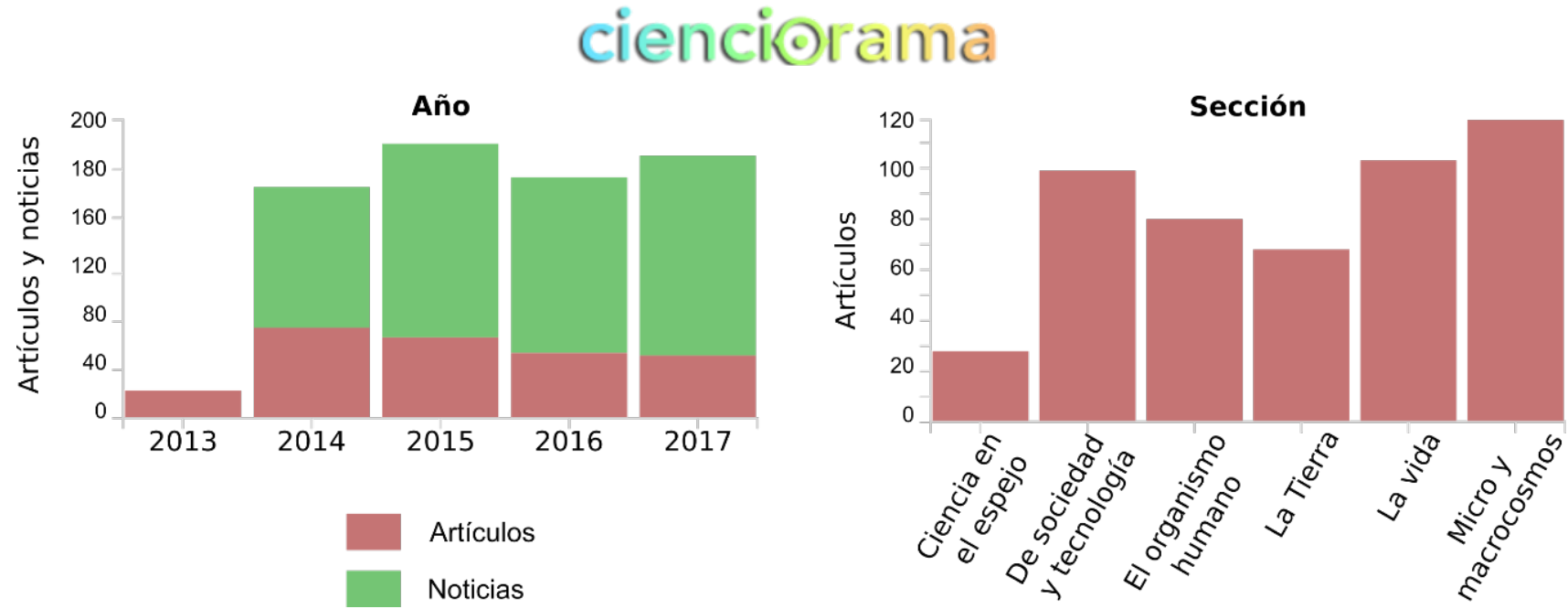


\section{Más allá de la redacción}

El Taller/Portal Cienciorama posibilita el trabajo personalizado y va mucho más allá de la redacción de textos, pues implica estimular la capacidad creativa, lograr una estructura narrativa y desarrollo argumental; hacer un uso adecuado y fundamentado de la información; alcanzar un lenguaje claro apoyado en material gráfico accesible y que en muchos casos es confeccionado ad hoc.

En el Taller/Portal Cienciorama pensamos que es muy importante democratizar el conocimiento y hacerlo accesible a quienes se pueden beneficiar de él, porque vivimos en sociedades en las que es una herramienta indispensable para vivir.

El lenguaje es el gran patrimonio de la humanidad, enriquecerlo es enriquecernos. Aunque la literatura en un sentido tradicional contribuye a ello, es necesario que en otros terrenos haya empeños literarios porque éstos implican siempre una mayor comunicación. La literatura es comunicación, es una lucha por ampliar el lenguaje para que exprese incluso lo más difícil de revelar.

En países como el nuestro, en los que el analfabetismo ha beneficiado tanto a una minoría con poder, es preciso combatirlo en todos los terrenos. En Cienciorama tratamos de promover la libertad y creatividad en el ámbito de una divulgación científica crítica. El taller presencial es un espacio de retroalimentación y discusión indispensable para los participantes que sería imposible en otras condiciones y fuera del ámbito universitario.

El Taller/Portal Cienciorama se encuentra en la Dirección General de Divulgación de la Ciencia de la UNAM y se puede acceder al sitio a través de las páginas de la UNAM y de la Sociedad Mexicana para la Divulgación de la Ciencia y la Técnica, misma que lo ha apoyado a lo largo de su historia.

Agradecemos a Mariana Esther Martínez su apoyo en la confección de las figuras.

\section{Referencias}

* Cruz, J., et al. (2015). Una aproximación semi-empírica, la calidad en el periodismo de ciencia, en Elaine Reynoso Haynes (coord.), Hacia dónde va la Ciencia en México. Comunicación Pública de la Ciencia. México: El Oficio II.

* Estrada, L. (2017). De cienciómanos e informantes, en Juan Tonda Coord. 30 años de divulgar la ciencia y la técnica. México: SOMEDICyT.

* Estrada, L. (2002). La divulgación de la ciencia, en Juan Tonda, Ana María Sánchez y Nemesio Chávez (coords.) Antología de la Divulgación de la ciencia en México. México: DGDC/UNAM. 
* López Beltrán, C. (2002). Fronteras: sobre el lenguaje común y el lenguaje científico, en Juan Tonda, Ana María Sánchez y Nemesio Chávez (coords.) Antología de la Divulgación de la ciencia en México. México: DGDC/UNAM

* Sánchez, A. M. (2000). La Divulgación de la ciencia como literatura, en Divulgación para divulgadores. México: DGDC/UNAM.

* Reynoso Haynes, E. (2008). La comunicación de la ciencia y la evaluación de programas para formar comunicadores, en Lozano, M. y C. Sánchez-Mora (coords.), Evaluando la comunicación de la ciencia: Una perspectiva latinoamericana. México: CYTED, AECI, DGDC-UNAM.

\section{Ejemplos de pláticas en Cienciorama:}

https://www.youtube.com/watch?v=-05-Pnz580

https://www.youtube.com/watch?v=nR3j-Y83joo

https://www.youtube.com/watch?v=EE7ZPT-ynho

https://www.youtube.com/watch?v=F7Tgra1pEMM\&t=83s

https://www.youtube.com/watch?v=twMi7i3/mU8

\section{Cursos sobre escritura en ciencia en universidades:}

http://sciwrite.mit.edu/

- https://www.theatlantic.com/education/archive/2014/12/how-scientists-are-learning-to-write/383685/

- http://blogs.plos.org/scicomm/2017/09/11/comscicon-science-communicationworkshops-for-graduate-students-by-graduate-students/

http://ece.duke.edu/about/news/science-and-storytelling-collide-phd-plus-program

- http://www.rackham.umich.edu/content/storytelling-stem-relate-workshop-developing-your-scientific-narrative

\section{Cómo citar este artículo}

* Cruz Wilson, L., García Bergua, A. y Zamarrón Garza, G (2018). Un taller literario y un portal para divulgar la ciencia: Cienciorama. Revista Digital Universitaria (RDU). Vol. 19, núm. 2 marzo-abril. DOl: http://doi.org/10.22201/codeic.16076079e.2018. v19n2.a9. 